\title{
Real World Dynamic Appearance Enhancement with Procam Feedback
}

\author{
Toshiyuki Amano* \\ Graduate School of Information Science \\ Nara Institute of Science and Technology
}

\author{
Hirokazu Kato ${ }^{\dagger}$ \\ Graduate School of Information Science \\ Nara Institute of Science and Technology
}
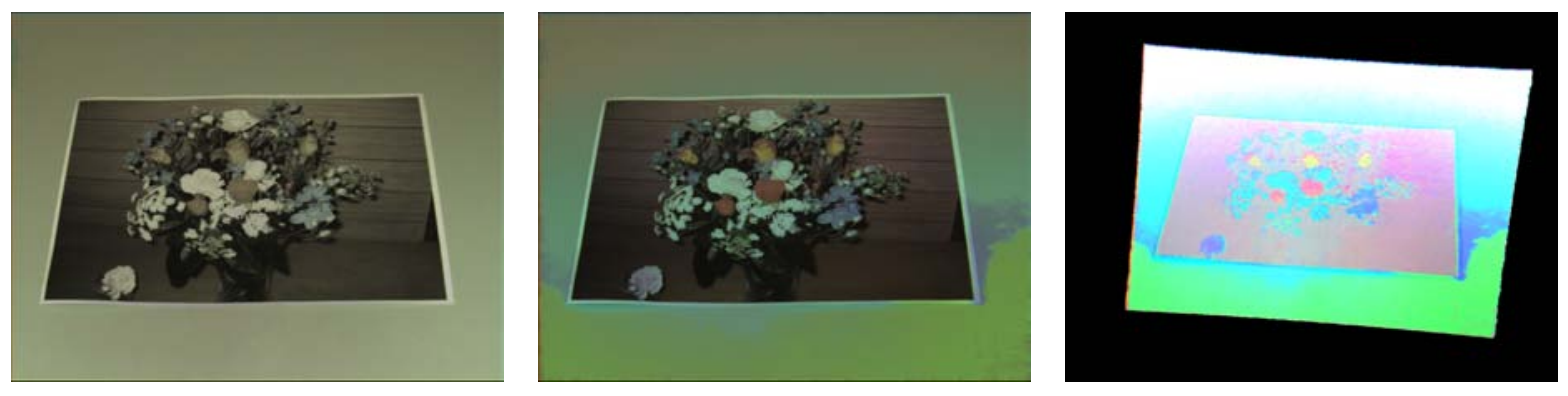

Figure 1: The contrast of a less saturated picture that exists in the real world is enhanced by our system. The left image is the appearance under the white light projection, the center image is enhanced result by the compensation light projection and the right image is compensation pattern that generated projector camera feedback. The enhancement is implemented by the dynamical feedback of projector camera system, and the system performed the enhancement with the processing speed of 11.4 fps under the fluorescent light condition.

\begin{abstract}
In this paper, a dynamic appearance enhancement method for the less saturated object is proposed. The appearance enhancement method proposed in this paper is realized with a projector camera dynamic feed back system. Therefore, the proposed method has an ability of the rapid and continuous appearance enhancement and it is useful for the human visual perception assistance. Also, the system not requires a pre-captured appearance of the target.
\end{abstract}

Keywords: Appearance, Enhancement, Procams Feedback

\section{Introduction}

In recent years, many adaptive projection systems that aim the photometric compensation for the non-uniformity surface color was proposed (e.g. [Grossberg et al. 2004; Fujii et al. 2005; Ashdown et al. 2006]).However not only photometric compensation but also opposite technique that enhances the non-uniform appearance of object is useful for human visual perception assistance. For example, visual assistance for the color-blind person and industrial visual inspection, color rendering control for stage lighting etc. Recently, Bimber [Bimber and Iwai 2008] proposed the technique that improves the dynamic range of the object's appearance by the superimposing with the projector. However, the continuous adaptation was not shown. Therefore, for the mentioned applications, we

*e-mail: amano@is.naist.jp

†e-mail: kato@is.naist.jp

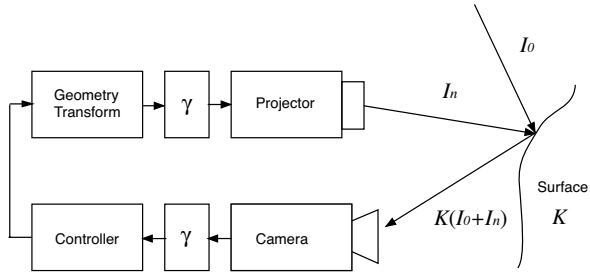

Figure 2: A Pro-Cam Feedback System.

adopt the dynamical projector camera feedback for the appearance enhancement that makes a point of rapid and continuous response to keep higher visibility without blinking for the visual perception assistance.

\section{Appearance enhancement with projector camera feedback}

The diagram of projector camera feedback system is shown in the figure 2. For Simplification, in this section we discuss about the feedback system without the geometrical transformation. In the conceptualistic expression, a received light energy by camera is in proportion to $\mathcal{K}\left(I_{0}+I_{n}\right)$, when compensation light $I_{n}$ is projected along with the environment light $I_{0}$ on the object's surface of reflection characteristics $\mathcal{K}$. From this relation, the illumination of $(n+1)$ th projection can be expressed by using the $n$th projection. In the initial condition, the first compensation light can be written as

$$
I_{1}=\mathcal{P}_{\text {prj }} \Gamma_{\text {prj }} \mathcal{G} \Gamma_{\text {cam }} \mathcal{P}_{\text {cam }} \mathcal{K} I_{0}=\mathcal{G}^{\prime} \mathcal{K} I_{0}
$$

Copyright $\odot 2008$ by the Association for Computing Machinery, Inc. Permission to make digital or hard copies of part or all of this work for personal or classroom use is granted without fee provided that copies are not made or distributed for commercial advantage and that copies bear this notice and the full citation on the first page. Copyrights for components of this work owned by others than ACM must be honored. Abstracting with credit is permitted. To copy otherwise, to republish, to post on servers, or to redistribute to lists, requires prior specific permission and/or a fee. Request permissions from Permissions Dept, ACM Inc., fax +1 (212) 869-0481 or e-mail permissions@acm.org.

PROCAMS 2008, Marina del Rey, California, August 10, 2008.

(C) 2008 ACM 978-1-60558-272-6/08/0008 $\$ 5.00$

where $\Gamma_{c a m}$ and $\Gamma_{p r j}$ are gamma curve and gamma correction, $\mathcal{P}_{\text {prj }}$ and $\mathcal{P}_{\text {cam }}$ are converting operators between illumination and pixel value of camera and projector respectively. The $\mathcal{G}$ is feedback operator that means feedback gain at the linear model, and $\mathcal{G}^{\prime}$ is feedback operator that includes all camera and projector properties. Since $(n-1)$ th compensation light is projected along with 
environment light $I_{0}$, the $n$th compensation light is

$$
I_{n}=\mathcal{G}^{\prime} \mathcal{K}\left(I_{0}+I_{n-1}\right)=\sum_{j=1}^{n}\left(\mathcal{G}^{\prime} \mathcal{K}\right)^{j} I_{0}
$$

From the result of computational simulation based on the equation 2, we adapted fractional feedback such as

$$
I_{*}^{\prime}=\frac{3 I_{*}}{I_{R}+I_{G}+I_{B}} I_{\max },
$$

to improve the convergence speed and avoid overflow of the output power. Where $* \in\{R, G, B\}$ is index of color channel and $I_{*}^{\prime}$ is the brightness expression of each color channel. The $I_{\max }$ means the maximum brightness level of $I_{*}$ (i.e. it is 255 if we use 8-bit image). In the following, we express the bright level by normalized value such as $I_{*} \in[0,1]$ and omit $I_{\max }$ because $I_{\max }=1$.

\subsection{Convergence Value Analysis}

The fractional feedback system is described in the linear matrix by using of the homogeneous expression $\mathbf{I}=\left(I_{R}, I_{G}, I_{B}, 1\right)^{T}$, and the output of projector is

$$
\left[\begin{array}{c}
I_{R}^{\prime \prime} \\
I_{G}^{\prime \prime} \\
I_{B}^{\prime \prime} \\
1
\end{array}\right] \sim\left[\begin{array}{cccc}
3 g & 0 & 0 & 0 \\
0 & 3 g & 0 & 0 \\
0 & 0 & 3 g & 0 \\
1 & 1 & 1 & 0
\end{array}\right]\left[\begin{array}{cc}
K_{3 \times 3} & \mathbf{0} \\
\mathbf{0} & 1
\end{array}\right]\left[\begin{array}{c}
I_{R} \\
I_{G} \\
I_{B} \\
1
\end{array}\right] .
$$

Where $K_{3 \times 3}$ is color mixing a matrix that includes reflection characteristics of the surface and chromatic dispersion between RGB channels of projector and camera.The $g$ is feedback gain and we put $\mathcal{G}^{\prime}$ as the simple gain control of each pixel. If we omit chromatic dispersion, the matrix $K_{3 \times 3}$ can be written as

$$
K_{3 \times 3}=\left[\begin{array}{ccc}
k_{R} & 0 & 0 \\
0 & k_{G} & 0 \\
0 & 0 & k_{B}
\end{array}\right] .
$$

Then, the final compensation light is

$$
\begin{aligned}
\mathbf{I}_{\infty}= & \left(\mathbf{1}-G^{\prime} K\right)^{-1} G^{\prime} K \mathbf{I}_{0} \\
= & {\left[\begin{array}{cccc}
\frac{3 g k_{R}}{1-3 g k_{R}} & 0 & 0 & 0 \\
0 & \frac{3 g k_{G}}{1-3 g k_{G}} & 0 & 0 \\
0 & 0 & \frac{3 g k_{B}}{1-3 g k_{B}} & 0 \\
\frac{k_{R}}{1-3 g k_{R}} & \frac{k_{G}}{1-3 g k_{G}} & \frac{k_{B}}{1-3 g k_{B}} & 0
\end{array}\right] \mathbf{I}_{0} . }
\end{aligned}
$$

From eq.(6), we can confirm that the brightness of final compensation light is converging according to the feedback gain and the system is stable. For example, if we apply this system to the white object such as $k_{R}=k_{G}=k_{B}$, the final compensation light is $\mathbf{I}_{\infty}=(g, g, g, 1)^{T}$, and in case of yellow object $k_{R}=k_{G}, k_{B}=0$, we get $\mathbf{I}_{\infty}=\left(\frac{3}{2} g, \frac{3}{2} g, 0,1\right)^{T}$ under the white environment light $\mathbf{I}_{0}$. Therefore, it was confirmed the feedback gain $g$ controls the brightness of compensation light that enhances the object appearance.

\subsection{Contrast Control}

We realized the brightness control of compensation light by using of fractional feedback. However, not only brightness but also the contrast control of the compensation light is effective for appearance enhancement. Therefore, we add the offset $t_{0} \in[0,1]$ and control the contrast of input image $\operatorname{Im}_{*}(x, y)$ by

$$
t_{*}(x, y)=\left(1-t_{0}\right) \operatorname{Im}_{*}(x, y)+t_{0}
$$

and we put the compensation light power as

$$
P_{*}(x, y)=\frac{3 g t_{*}(x, y)}{t_{R}(x, y)+t_{G}(x, y)+t_{B}(x, y)}
$$

where $x, y$ are the coordinate of image surface of camera and projector, and input image is normalized to $\operatorname{Im}(x, y) \in[0,1]$. At the implementation, since the brightness of compensation light $P_{*}(x, y)$ is normalized, we give the projection output $255 \times$ $P_{*}(x, y)$ if the gradation of the projector is 8-bit.

\section{Experimental Results}

The camera (SONY XCD-710CR, RGB 8bit, $1024 \times 768$ resolution, $\gamma=1$ ) is attached with projector (CANON LV7210, RGB 8bit, $1024 \times 768$ resolution) and the compensation light is projected at a slant from above under the fluorescent light. At the first, we calibrated the pixel correspondence between acquired image and projection image by the projection of gray code images. Next, we calibrated color space between projector and camera. The figure 1 shown the enhancement results for less saturated pictures with the parameters $g=0.8, t_{0}=0.05$. We can confirm the contrast of less saturated picture was improved by the proposed method. The compensation pattern that has been colored with same color as the original appearance on the corresponded parts was generated that reflected the original picture appearance. This enhancement was executed sequentially at a speed of $11.4 \mathrm{fps}$ by the Core2Duo $2.4 \mathrm{GHz}$ PC (Apple MacBookPro), and the compensation image pattern was stable within 5 steps for static scene. Therefore, the response time by our experimental equipment was $0.44 \mathrm{sec}$.

\section{Conclusion}

In this paper, we proposed a appearance enhancement method by the projector camera feedback system. This enabled the enhancements of object's brightness and contrast in sequentially at the real world and its effectiveness was confirmed by experimental results of less saturated picture and faded picture. The remaining problems are the color compensation non-uniformity that caused lens aberration and improvement for the brightness contrast for the faded picture. We would like to solve these problems in a future work.

\section{References}

Ashdown, M., OKabe, T., SATo, I., And SATo, Y. 2006. Robust content-dependent photometric projector compensation. In CVPRW '06: Proceedings of the 2006 Conference on Computer Vision and Pattern Recognition Workshop, IEEE Computer Society, Washington, DC, USA, 6.

Bimber, O., AND IWAI, D. 2008. Superimposing dynamic range. Technical Report 1358, Bauhaus-University Weimar, Germany.

Fujit, K., Grossberg, M. D., And Nayar, S. K. 2005. A projector-camera system with real-time photometric adaptation for dynamic environments. In CVPR '05: Proceedings of the 2005 IEEE Computer Society Conference on Computer Vision and Pattern Recognition (CVPR'05) - Volume 1, IEEE Computer Society, Washington, DC, USA, 814-821.

Grossberg, M. D., Peri, H., Nayar, S. K., AND BelHUMEUR, P. N. 2004. Making one object look like another: Controlling appearance using a projector-camera system. cvpr $01,452-459$. 